\title{
Study of properties of solutions for quasilinear parabolic systems
}

\author{
Yunzhu Gao, Qiu Meng and Yingjia Guo \\ Department of Mathematics and Statistics, Beihua University, Jilin City, PR China
}

\begin{abstract}
In this paper, we concern with degenerate and quasilinear parabolic systems not in divergence form with null Dirichlet boundary conditions and positive initial conditions. The local existence and uniqueness of classical solution are proved. Moreover, It will be proved that all solutions exist globally with homogeneous Dirichlet boundary condition.
\end{abstract}

\section{Introduction}

In this paper, we consider the following degenerate and quasilinear parabolic systems not in divergence form: $\left\{\begin{array}{l}u_{i t}=u_{i}^{p_{i}}\left(\Delta u_{i}+a_{i} u_{i+1}\right), i=1,2, \cdots, m, u_{m+1}:=u_{1} \\ u_{i}(x, 0)=u_{i 0}(x), i=1,2, \cdots, m, x \in \Omega \\ u_{i}(x, t)=0, i=1,2, \cdots, m, x \in \partial \Omega, t>0\end{array}\right.$

where $a_{i}, p_{i}$, are positive constants, $\Omega \in R^{N}$ is a bounded domain with smooth boundary $\partial \Omega$, The initial data $u_{i 0}(x), \quad i=1,2, \cdots, m$, satisfy

$\left\{\begin{array}{l}u_{i 0}(x) \in C^{1}(\bar{\Omega}), u_{i 0}(x)>0, i=1,2, \cdots, m, \text { in } \Omega, \\ u_{i}(x, t)=0, \frac{\partial u_{i 0}}{\partial \eta}<0, i=1,2, \cdots, m, \text { in } \partial \Omega,\end{array}\right.$

here $\eta$ is the outward normal vector on $\partial \Omega$. The first aim of this paper is to prove the local existence and uniqueness of positive classical solutions. The first aim of this paper is to prove the local existence and uniqueness of positive classical solution of (1) exist globally if and only if $\prod_{i=1}^{m} a_{i} \leq \lambda_{1}^{m}$, where $\lambda_{1}$ is the first eigenvalue of $-\Delta$ in $\Omega$ with homogeneous Dirichlet boundary condition.

Throughout this paper we say that $\left(u_{1}, u_{2}, \cdots, u_{m}\right)$ is a classical solution of a initial and boundary value problem

$\left(u_{1}, u_{2}, \cdots, u_{m}\right) \in C^{2,1}(\Omega \times(0, T)) \cap C(\bar{\Omega} \times[0, T))$

for some $T: 0 \leq T<+\infty$, and $\left(u_{1}, u_{2}, \cdots, u_{m}\right)$ satisfies the differential equations in $\Omega \times(0, T)$ and the initial and boundary conditions continuously of this problem.

This system can be used to describe the development of multiple groups in the dynamics of biological groups where $u_{i}, i=1,2, \cdots, m$ are the densities of the different groups.

Our paper is organized as follows. In Section 2 we prove the local existence, uniqueness and comparison principle of solution to (1). In Section 3 we will prove that the solutions of (1) blows up in finite time if $\Pi_{i=1}^{m} a_{i} \geq \lambda_{1}^{m}$ and finally we prove that if $\Pi_{i=1}^{m} a_{i} \leq \lambda_{1}^{m}$ then the solution of (1) exists globally.

\section{Existence, uniqueness of solutions}

In this section, we shall first consider local existence of system (1). Since $u_{i}, i=1,2, \cdots, m$ on the boundary $\partial \Omega$, the equation of (1) is not strictly parabolic type. The standard parabolic theory [1,2] cannot be used directly to prove the local existence of solution to problem (1). To overcome this difficulty, we will use the standard approximate method, see [3], For $\varepsilon>0$, considering the following approximate problem

$\left\{\begin{array}{l}u_{i \varepsilon t}=f_{i \varepsilon}\left(u_{i \varepsilon}\right)\left(\Delta u_{i \varepsilon}+a_{i} u_{i+1 \varepsilon}, i=1,2, \cdots, m, x \in \Omega, t>0\right. \\ u_{i \varepsilon}(x, 0)=u_{i 0}(x)+\varepsilon, i=1,2, \cdots, m, x \in \Omega \\ u_{i \varepsilon}(x, t)=\varepsilon, i=1,2, \cdots, m, x \in \partial \Omega, t>0\end{array}\right.$

Where $u_{m+1}:=u_{1}, f_{i \varepsilon}, i=1,2, \cdots, m$ are smooth function, $f_{i \varepsilon} \geq\left(\frac{\varepsilon}{2}\right)^{p_{i}}, \quad i=1,2, \cdots, m$

$$
f_{i \varepsilon}=\left\{\begin{array}{cl}
u_{i}^{p_{i}} & , u_{i} \geq \varepsilon \\
\left(\frac{\varepsilon}{2}\right)^{p_{i}} & , u_{i}<\varepsilon
\end{array}\right.
$$

The standard parabolic theory shows that (3) admits a unique maximal defined classical solution 
$\left(u_{1 \varepsilon}, u_{2 \varepsilon}, \cdots, u_{m \varepsilon}\right)$ which is defined on $[0, T(\varepsilon))$ here $.0<T(\varepsilon) \leq \infty$.The maximum principle implies that $\quad u_{i \varepsilon} \geq \varepsilon \quad, \quad i=1,2, \cdots, m \quad$ which gives $f_{i \varepsilon}\left(u_{i \varepsilon}\right) \geq u_{i \varepsilon}^{p i}$ and hence $\left(u_{1 \varepsilon}, u_{2 \varepsilon}, \cdots, u_{m \varepsilon}\right)$ the following problem

$$
\left\{\begin{array}{l}
u_{i \varepsilon t}=u_{i \varepsilon}^{p i}\left(\Delta u_{i \varepsilon}+a_{i} u_{i+1 \varepsilon}\right), x \in \Omega, 0<t<T(\varepsilon), \\
u_{i \varepsilon}(x, 0)=u_{i 0}(x)+\varepsilon, x \in \Omega \\
u_{i \varepsilon}(x, t)=\varepsilon, x \in \partial \Omega, 0<t<T(\varepsilon)
\end{array}\right.
$$

Where $i=1,2, \cdots, m, \quad u_{m+1} \geq u_{1}$. The fact that $u_{i \varepsilon} \geq \varepsilon$ shows that problem (4) is not degenerate. Since problem (4) is quasimonotone increasing in $\left(u_{1 \varepsilon}, u_{2 \varepsilon}, \cdots, u_{m \varepsilon}\right)$, applying the comparison principle for parabolic systems (.[1, Chapter IV]) we have the following two lemmas.

Lemma $2.1 \quad$ Assume that positive functions $u_{i} \in C\left(\bar{\Omega} \times[0, T(\varepsilon)) \cap C^{2,1}(\Omega \times(0, T(\varepsilon)))\right.$ $, i=1,2, \cdots, m$. If $\left(u_{1}, u_{2}, \cdots, u_{m}\right)$ is a lower (or upper) solution of

Then $\quad\left(u_{1}, u_{2}, \cdots, u_{m}\right) \leq(\geq)\left(u_{1 \varepsilon}, u_{2 \varepsilon}, \cdots, u_{m \varepsilon}\right) \quad$ on $\bar{\Omega} \times[0, T(\varepsilon)$.

Lemma 2.2 Let $\varepsilon_{1}<\varepsilon_{2}$ then $T\left(\varepsilon_{1}\right)<T\left(\varepsilon_{2}\right)$ and $\left(u_{1 \varepsilon_{1}}, u_{2 \varepsilon_{1}}, \cdots, u_{m \varepsilon_{1}}\right) \quad<\left(u_{1 \varepsilon_{2}}, u_{2 \varepsilon_{2}}, \cdots, u_{m \varepsilon_{2}}\right) \quad$ on $\bar{\Omega} \times\left[0, T\left(\varepsilon_{2}\right)\right.$.

It follows from Lemma 2.2 that there exist $T: 0<T \leq \infty \quad$ and $\quad\left(u_{1}(x, t), u_{2}(x, t), \cdots u_{m}(x, t)\right)$ which is defined on $\bar{\Omega} \times[0, T)$ such that $T(\varepsilon) \quad, \quad T=\sup _{\varepsilon>0} T(\varepsilon) \quad$ and $\left(u_{1 \varepsilon}(x, t), u_{2 \varepsilon}(x, t), \cdots u_{m \varepsilon}(x, t)\right) \rightarrow$ $\left(u_{1}(x, t), u_{2}(x, t), \cdots u_{m}(x, t)\right)$ as $\varepsilon \rightarrow 0$.

To discuss the positivity and regularity of $\left(u_{1}(x, t), u_{2}(x, t), \cdots u_{m}(x, t)\right)$, we should give some estimate of the lower bound of $\left(u_{1}(x, t), u_{2}(x, t), \cdots u_{m}(x, t)\right) \quad$. Let $\quad \lambda_{1} \quad$ and $\varphi(x)>0(x \in \Omega)$ be respectively the first eigenvalue and the corresponding eigenfunction of the following eigenvalue problem

$$
-\Delta \varphi=\lambda \varphi \text { in } \Omega, \varphi=0 \text { on } \partial \Omega \text {. }
$$

and think that $\max _{\bar{\Omega}} \varphi(x)=1$. Then $\lambda_{1}>0$ and $\frac{\partial \varphi}{\partial \eta}<0$ on $\partial \Omega$.

By the assumptions on $\left(u_{10}, u_{20}, \cdots u_{m 0}\right)$ we see that there exist positive constants $k_{i}, i=1,2, \cdots, m$ such that

$$
\left\{\begin{array}{l}
u_{i 0}(x) \geq k_{i} \varphi(x), i=1,2, \cdots m, x \in \bar{\Omega}, \\
\frac{\lambda_{1}}{a_{i}} \leq \frac{k_{i+1}}{k_{i}}, i=1,2, \cdots m, k_{m+1}:=k_{m}, \text { if } \prod_{i=1}^{m} a_{i} \geq \lambda_{1}^{m}, \\
\frac{\lambda_{1}}{a_{i}}>\frac{k_{i+1}}{k_{i}}, i=1,2, \cdots m, k_{m+1}:=k_{m}, \text { if } \prod_{i=1}^{m} a_{i}<\lambda_{1}^{m},
\end{array}\right.
$$

Lemma 2.3 Let $\varepsilon<1, \quad\left(u_{1 \varepsilon}, u_{2 \varepsilon}, \cdots u_{m \varepsilon}\right)$ be the solution of (4), and positive constants $k_{1}, k_{2}, \cdots k_{m}$ satisfy

(5). We have the followingestimates:

(i) If $\prod_{i=1}^{m} a_{i} \geq \lambda_{1}^{m}$, then for $i=1,2, \cdots, m$, $u_{i \varepsilon}(x, t) a_{i} \geq k_{i} \varphi(x)+\varepsilon, \quad \forall(x, t) \in \bar{\Omega} \times[0, T(\varepsilon))$,

(ii) If $\prod_{i=1}^{m} a_{i} \leq \lambda_{1}^{m}$, then for $i=1,2, \cdots, m$, $u_{i \varepsilon}(x, t) a_{i} \geq k_{i} \varphi(x) e^{-\mu t}+\varepsilon, \quad \forall(x, t) \in \bar{\Omega} \times[0, T(\varepsilon))$ where

$\mu \geq \max \left\{\left(1+k_{i}\right)^{p_{i}}\left(\lambda_{1}-a_{i} \frac{k_{i+1}}{k_{i}}\right), i=1,2, \cdots, m, k_{m+1}:=k_{1}\right\}$

Proof: (i) When $\prod_{i=1}^{m} a_{i} \geq \lambda_{1}^{m}$, set $u_{i \varepsilon}=k_{i} \varphi(x)+\varepsilon, i=1,2, \cdots, m$, In view of (5), for $u_{m+1}:=u_{1}$ and, $k_{m+1}:=k_{1}$ we have,

$\underline{u}_{i \varepsilon}^{p_{i}}\left(\underline{u}_{i \varepsilon}+a_{i} \underline{u}_{i+1 \varepsilon}\right)=\left(k_{i} \varphi+\varepsilon\right)^{p_{i}}\left(-\lambda_{1} k_{i} \varphi+a_{i}\left(k_{i+1} \varphi+\varepsilon\right)\right)$

$>\left(k_{i} \varphi+\varepsilon\right)^{p_{i}}\left(a_{i} k_{i+1}-\lambda_{i} k_{i}\right) \varphi$

$\geq 0=\underline{u}_{i s t}, \quad(x, t) \in \Omega \times(0, T(\varepsilon))$,

$\underline{u}_{i \varepsilon}(x, t)=\varepsilon, \quad(x, t) \in \partial \Omega \times(0, T(\varepsilon))$

$\underline{u}_{i \varepsilon}(x, 0) \leq \underline{u}_{i \varepsilon}(x, 0), \quad x \in \bar{\Omega}$.

This shows that $\left(\underline{u}_{1 \varepsilon}, \underline{u}_{2 \varepsilon}, \cdots \underline{u}_{m \varepsilon}\right)$ is a lower solution of (4). Lemma 2.1 implies that (6) holds.

When $\quad \prod_{i=1}^{m} a_{i}<\lambda_{1}^{m} \quad$ set $\underline{u}_{i \varepsilon}(x, t)=k_{i} \varphi(x) e^{-\mu t}+\varepsilon, i=1,2, \cdots, m$ Then from (5) and (7), for $u_{m+1}:=u_{1}$ and $k_{m+1}:=k_{1}$ we have,

$\underline{u}_{i \varepsilon t}=-\mu k_{i} \varphi e^{-\mu t}$,

$\underline{u}_{i \varepsilon}^{p_{i}}\left(\Delta u_{i \varepsilon}+a_{i} \underline{u}_{i+1 \varepsilon}\right)$

$=\left(k_{i} \varphi e^{-\mu t}+\varepsilon\right)^{p_{i}}\left(-k_{i} \lambda_{1} \varphi e^{-\mu t}+a_{i} k_{i+1} \varphi e^{-\mu t}+a_{i} \varepsilon\right)$

$>\left(k_{i} \varphi e^{-\mu t}+\varepsilon\right)^{p_{i}}\left(a_{i} k_{i+1}-k_{i} \lambda_{1}\right) \varphi e^{-\mu t}$

$\geq\left(\varepsilon+k_{i}\right)^{p_{i}}\left(a_{i} k_{i+1}-k_{i} \lambda_{1}\right) \varphi e^{-\mu t}$

$>\left(1+k_{i}\right)^{p_{i}}\left(a_{i} k_{i+1}-k_{i} \lambda_{1}\right) \varphi e^{-\mu t}$

$\geq-\mu k_{i} \varphi e^{-\mu t}=\underline{u}_{i c t}, \quad(x, t) \in \Omega \times(0, T(\Omega))$ 


$$
\begin{aligned}
& \underline{u}_{i \varepsilon}(x, t)=\varepsilon, \quad(x, t) \in \partial \Omega \times(0, T(\varepsilon)), \\
& \underline{u}_{i \varepsilon}(x, 0) \leq u_{i \varepsilon}(x, 0), \quad x \in \bar{\Omega} .
\end{aligned}
$$

The same reason as that of (i) shows that (6) holds. The proof is completed.

Theorem 2.1 Problem (1) has a positive classical solution

$\left(u_{1}, u_{2}, \cdots u_{m}\right) \in\left[C^{2+\beta, 1+\beta / 2}(\Omega \times(0, T)) \cap C(\bar{\Omega} \times[0, T))\right]$ for some $\beta: 0<\beta<1$, and $T: 0<T \leq \infty$.

Proof: Lemma 2.3 shows that $\left(u_{1}, u_{2}, \cdots u_{m}\right)$, the limit of $\left(u_{1 \varepsilon}, u_{2 \varepsilon}, \cdots u_{m \varepsilon}\right)$, is positive in $\Omega \times(0, T)$. In view of Lemma 2.2 and Lemma 2.3, the classical results of local Schauder estimates (see[4]) imply that $u_{i \varepsilon} \in C^{2+\alpha,(1+\alpha) / 2}(\Omega \times(0, T(\varepsilon))$ for some $\alpha>0$.

Furthermore, for any $\Omega_{0} \subset \Omega$ and $0<\tau<T_{0}<T$, we have

$$
\begin{gathered}
\left(u_{1 \varepsilon}, u_{2 \varepsilon}, \cdots u_{m \varepsilon}\right) \rightarrow\left(u_{1}, u_{2}, \cdots u_{m}\right) \text { in } \\
{\left[C^{2+\beta, 1+\beta / 2}\left(\bar{\Omega}_{0} \times\left[\tau, T_{0}\right]\right)\right]^{m}, 0<\beta<\alpha, \varepsilon \rightarrow 0^{+} .}
\end{gathered}
$$$$
\text { Therefore, } \quad\left(u_{1}, u_{2}, \cdots u_{m}\right) \quad \text { belongs to }
$$
$C^{2+\beta, 1+\beta / 2}(\Omega \times(0, T(\varepsilon))$ and satisfies the differential equations of (1) in $\Omega \times(0, T)$. Fix $\varepsilon_{0}: 0<\varepsilon_{0}<1$. For any $\Omega_{0} \subset \Omega$ and $0<\varepsilon<\varepsilon_{0}$, From Lemma 2.1, we have $\quad \underline{u}_{i \varepsilon}(x, t)<u_{i \varepsilon_{0}}(x, t), i=1,2, \cdots, m \quad$ on $\bar{\Omega}_{0} \times\left[0, T\left(\varepsilon_{0}\right) / 2\right]$, in view of Lemma 2.3 , the $L^{p}$ theory and embedding theorem show that the $C^{\alpha, \alpha / 2}\left(\bar{\Omega}_{0} \times\left[0, T\left(\varepsilon_{0}\right) / 2\right]\right) \quad$ norm of $u_{i \varepsilon}, i=1,2, \cdots, m$ is uniformly

bounded above for all $\varepsilon<\varepsilon_{0}$. Hence

$$
\begin{aligned}
& \left(u_{1 \varepsilon}, u_{2 \varepsilon}, \cdots u_{m \varepsilon}\right) \rightarrow\left(u_{1}, u_{2}, \cdots u_{m}\right) \text { in } \\
& {\left[C^{\beta, \beta / 2}\left(\bar{\Omega}_{0} \times\left[0, T\left(\varepsilon_{0}\right) / 2\right]\right)\right]^{m}, 0<\beta<\alpha, \varepsilon \rightarrow 0^{+},}
\end{aligned}
$$

which implies that $u_{i} \in C(\Omega \times[0, T]), i-1,2, \cdots, m$. Similar to the arguments of $[4,5,6]$ we can prove that $\left(u_{1}, u_{2}, \cdots u_{m}\right)$ is continuous on $\partial \Omega \times(0, T)$. Using the initial and boundary conditions of (4) we see that $\left(u_{1}, u_{2}, \cdots u_{m}\right)$ satisfies the initial and boundary conditions of (1) , i.e. $\left(u_{1}, u_{2}, \cdots u_{m}\right) \in\left[C^{2+\beta, 1+\beta / 2}(\Omega \times(0, T) \cap C[0, T))\right]^{m}$ is a classical solution of (1). The proof is completed. Next, we will prove the uniqueness of positive classical solution of (1).

Theorem 2.2 Problem (1) has a unique positive classical solution

$$
\left(u_{1}, u_{2}, \cdots u_{m}\right) \in\left[C^{2+\beta, 1+\beta / 2}(\Omega \times(0, T) \cap C(\overline{\Omega \times}[0, T))]^{m}\right.
$$$$
\text { , for some } \beta: 0<\beta<1 \text { and } T: 0<T \leq \infty \text {. }
$$

Moreover, if $T<\infty$ then $\varlimsup_{t \rightarrow T^{-}} \max _{\bar{\Omega}} u(\cdot, t)=\infty$
Proof: Suppose that $\left(v_{1}, v_{2}, \cdots v_{m}\right)$ is also a positive classical solution of (1). By Lemma 2.1 we have that $v_{i}<u_{i \varepsilon}, i=1,2, \cdots, m \quad$ and hence $v_{i}<u_{i}, i=1,2, \cdots, m$. For $\delta>0$ sufficiently small, denote $\Omega_{\delta}=\{x \in \Omega \mid \operatorname{dist}(x, \partial \Omega) \geq \delta\}$. Let $\lambda_{\delta}$ and ]$^{m} \varphi_{\delta}(x)>0$ be respectively the first eigenvalue and the corresponding eigenfunction of $-\Delta$ in $\Omega_{\delta}$ with homogeneous boundary condition, and think that $\max _{\Omega_{\delta}} \varphi_{\delta}=1$. Multiplying the equations of (1) $\varphi_{\delta} / u_{i}^{p_{i}}, i=1,2, \cdots, m$ respectively, and integrating the results by parts we have

$$
\begin{aligned}
& \int_{\Omega_{\delta}} \xi_{i}\left(u_{i}\right) \varphi_{\delta} d x=\int_{\Omega_{\delta}} \xi_{i}\left(u_{i 0}\right) \varphi_{\delta} d x-\int_{0}^{t} \int_{\partial \Omega_{\delta}} u_{i} \frac{\partial \varphi_{\delta}}{\partial \eta} d S_{x} d t \\
& +a_{i} \int_{0}^{t} \int_{\Omega_{\delta}} u_{i+1} \varphi_{\delta} d x d t-\lambda_{\delta} \int_{0}^{t} \int_{\Omega_{\delta}} u_{i} \varphi_{\delta} d x d t
\end{aligned}
$$

where $\xi_{i}\left(u_{i}\right)=\left\{\begin{array}{l}\frac{u_{i}^{1-p_{i}}}{1-p_{i}}, \text { if } p_{i} \neq 1, \quad i=1,2, \cdots, m, \\ \text { Inu }_{i}, \text { if } p_{i}=1,\end{array}\right.$ $u_{m+1}:=u_{1}$.

Similarly

$$
\begin{gathered}
\int_{\Omega_{\delta}} \xi_{i}\left(v_{i}\right) \varphi_{\delta} d x=\int_{\Omega_{\delta}} \xi_{i}\left(u_{i 0}\right) \varphi_{\delta} d x-\int_{0}^{t} \int_{\partial \Omega_{\delta}} v_{i} \frac{\partial \varphi_{\delta}}{\partial \eta} d S_{x} d t \\
+a_{i} \int_{0}^{t} \int_{\Omega_{\delta}} v_{i+1} \varphi_{\delta} d x d t-\lambda_{\delta} \int_{0}^{t} \int_{\Omega_{\delta}} v_{i} \varphi_{\delta} d x d t
\end{gathered}
$$

where $i=1,2, \cdots, m, v_{m+1}:=v_{1}$. Then (8) subtract (9) respectively, we have

$$
\begin{gathered}
\int_{\Omega_{\delta}}\left[\xi_{i}\left(u_{i}\right)-\xi\left(v_{i}\right)\right] \varphi_{\delta} d x=-\int_{0}^{t} \int_{\partial \Omega_{\delta}}\left(u_{i}-v_{i}\right) \frac{\partial \varphi_{\delta}}{\partial \eta} d S_{x} d t \\
+a_{i} \int_{0}^{t} \int_{\Omega_{\delta}}\left(u_{i+1}-v_{i+1}\right) \varphi_{\delta} d x d t \\
-\lambda_{\delta} \int_{0}^{t} \int_{\Omega_{\delta}}\left(u_{i}-v_{i}\right) \varphi_{\delta} d x d t
\end{gathered}
$$

For any given $T_{0}: 0<T_{0}<T, \quad$ denot $M=\max _{\bar{\Omega} \times\left[0, T_{0}\right]}\left(\sum_{i=1}^{m} u_{i}\right) \quad$. Applying $v_{i}<u_{i}, i=1,2, \cdots, m \quad$ we get that $\int_{\Omega_{\delta}}\left[\xi_{i}\left(u_{i}\right)-\xi_{i}\left(v_{i}\right)\right] \varphi_{\delta} d x$

$$
=\int_{\Omega_{\delta}}\left(\int_{0}^{1} \xi_{i}^{\prime}\left(v_{i}+s\left(u_{i}-v_{i}\right)\right) d s\right)\left(u_{i}-v_{i}\right) \varphi_{\delta} d x
$$




$$
\begin{gathered}
=\int_{\Omega_{\delta}}\left(\int_{0}^{1}\left(v_{i}+s\left(u_{i}-v_{i}\right)\right)^{-p_{i}} d s\right)\left(u_{i}-v_{i}\right) \varphi_{\delta} d x \\
\geq M^{-p_{i}} \int_{\Omega_{\delta}}\left(u_{i}-v_{i}\right) \varphi_{\delta} d x
\end{gathered}
$$

From (10) and (11) it follows that

$$
\begin{gathered}
\int_{\Omega_{\delta}}\left[\sum_{i=1}^{m}\left(u_{i}-v_{i}\right)\right] \varphi_{\delta} d x \\
\leq-M_{1} \int_{0}^{t} \int_{\partial \Omega_{\delta}} \sum_{i=1}^{m}\left(u_{i}-v_{i}\right) \frac{\partial \varphi_{\delta}}{\partial \eta} d S_{x} d t \\
+M_{2} \int_{\Omega_{\delta}}\left[\sum_{i=1}^{m}\left(u_{i}-v_{i}\right)\right] \varphi_{\delta} d x d t,
\end{gathered}
$$

where $\quad M_{1}=\max \left\{M^{p_{i}}, i=1,2, \cdots, m\right\}$, $M_{2}=\sum_{i=1}^{m} M^{p_{i}}\left(a_{i}+\lambda_{\delta}\right)$. Applying Gronwall's lemma and then taking the limit as $\delta \rightarrow 0$ it follows that $u_{i} \equiv v_{i}, i=1,2, \cdots, m$. The proof is completed.

Finally we will give the comparison principle without proof for Problem (1).

$$
\begin{array}{lrr}
\text { Theorem } & \text { Assume } & \text { that } \\
\left(\bar{u}_{10}(x), \bar{u}_{20}(x), \cdots, \bar{u}_{m 0}(x)\right) & & \text { and } \\
\left(\underline{u}_{10}(x), \underline{u}_{20}(x), \cdots, \underline{u}_{m 0}(x)\right) \text { are continuous positive } \\
\text { functions in } \quad \text { and satisfy } \quad \text { (2). r Let } \\
\left(\bar{u}_{1}(x), \bar{u}_{2}(x), \cdots, \bar{u}_{m}(x)\right) & \text { and } \\
\left(\underline{u}_{1}(x), \underline{u}_{2}(x), \cdots, \underline{u}_{m}(x)\right) \text { be the positivesolutions of (1) }
\end{array}
$$
with initial data $\left(\bar{u}_{10}(x), \bar{u}_{20}(x), \cdots, \bar{u}_{m 0}(x)\right)$ and $\left(\underline{u}_{10}(x), \underline{u}_{20}(x), \cdots, \underline{u}_{m 0}(x)\right)$, respectively. Denote by $\bar{T}$ and $\underline{T}$ their maximal existence time, If $\left(\bar{u}_{10}(x), \bar{u}_{20}(x), \cdots, \bar{u}_{m 0}(x)\right)$

$\geq\left(\underline{u}_{10}(x), \underline{u}_{20}(x), \cdots, \underline{u}_{m 0}(x)\right)$ in $\Omega$, then $\bar{T} \leq \underline{T}$ and

$$
\begin{array}{r}
\left(\bar{u}_{1}(x), \bar{u}_{2}(x), \cdots, \bar{u}_{m}(x)\right) \geq\left(\underline{u}_{1}(x), \underline{u}_{2}(x), \cdots, \underline{u}_{m}(x)\right), \\
\forall(x, t) \in \bar{\Omega} \times[0, \bar{T}) .
\end{array}
$$

\section{Acknowledgements}

This work was financially supported by the National Natural Science Foundation of Tianyuan Youth Fund Project (11526035).

\section{References}

1. O.A. Ladyzenskaja, V.A. Solonnikov, N.N. Ural'ceva, Linear and Quasilinear Equations of Parabolic Type, Amer. Math. society, Providence, RI, 1968..
2. C.V. Pao, Nonlinear Parabolic and Elliptic Equations, Plenum, New York, 1992. J. Rauch. Discontinuous semilinear differential equations and multiple valued maps, Proc. Amer. Math. Soc. 64 (1977) 277-282.

3. S. Wang, M.X. Wang, C.H. Xie, A nonlinear degenerate diffusion equation not in divergence form, Z. Angew. Math. Phys. 51 (2000) 149-159.

4. A. Friedman, J.B. McLeod, Blow up of solutions of nonlinear degenerate parabolic equations, Arch. Rational Mech. Anal. 96 (1987) 55-80.

5. M. Wiegner, Blow-up for solutions of some degenerate parabolic equations, Differential Integral Equations 7 (1994) 1641-1647.

6. Y. Gao, W. Gao Existence and blow-up of solutions for a porous medium equation with nonlocal boundary condition. Applicable Analysis , 90(5)(2011), 799-809. 